TITLE:

\title{
Hydrothermal reactions of formaldehyde and formic acid: Free-energy analysis of equilibrium
}

\author{
$\operatorname{AUTHOR}(\mathrm{S})$ :
}

Matubayasi, N; Nakahara, M

\section{CITATION:}

Matubayasi, N ... [et al]. Hydrothermal reactions of formaldehyde and formic acid: Freeenergy analysis of equilibrium. JOURNAL OF CHEMICAL PHYSICS 2005, 122(7): 074509.

\section{ISSUE DATE:}

2005-02-15

URL:

http://hdl.handle.net/2433/50355

\section{RIGHT:}

Copyright 2005 American Institute of Physics. This article may be downloaded for personal use only. Any other use requires prior permission of the author and the American Institute of Physics. 


\title{
Hydrothermal reactions of formaldehyde and formic acid: Free-energy analysis of equilibrium
}

\author{
Nobuyuki Matubayasi ${ }^{\mathrm{a})}$ and Masaru Nakahara \\ Institute for Chemical Research, Kyoto University, Uji, Kyoto 611-0011, Japan
}

(Received 2 November 2004; accepted 23 November 2004; published online 10 February 2005)

\begin{abstract}
The chemical equilibria concerning formaldehyde and formic acid are computationally investigated in water over a wide range of thermodynamic conditions. The free energy is evaluated in the method of energy representation for the solvent effect on the decomposition processes of these two compounds. The solvation is found to suppress the production of nonpolar species from a polar. In the two competitive decomposition reactions of formic acid, the solvent strongly inhibits the decarboxylation $\left(\mathrm{HCOOH} \rightarrow \mathrm{CO}_{2}+\mathrm{H}_{2}\right)$ and its effect is relatively weak for the decarbonylation $\left(\mathrm{HCOOH} \rightarrow \mathrm{CO}+\mathrm{H}_{2} \mathrm{O}\right)$. The equilibrium weights for the two decomposition pathways of formic acid are determined by the equilibrium constant of the water-gas-shift reaction $\left(\mathrm{CO}+\mathrm{H}_{2} \mathrm{O} \rightarrow \mathrm{CO}_{2}\right.$ $+\mathrm{H}_{2}$ ), which is an essential and useful process in fuel technology. The reaction control by the solvent is then examined for the water-gas-shift reaction. Through the comparison of the equilibrium constants in the absence and presence of solvent, even the favorable side of the reaction is shown to be tuned by the solvent density and temperature. The reaction equilibrium is further treated for aldehyde disproportionation reactions involving formaldehyde and formic acid. The disproportionation reactions are found to be subject to relatively weak solvent effects and to be dominated by the electronic contribution. (C) 2005 American Institute of Physics.
\end{abstract}

[DOI: $10.1063 / 1.1849165$ ]

\section{INTRODUCTION}

Water is potentially a useful medium for organic chemical reactions. ${ }^{1,2}$ At ambient conditions, however, the utility of water as a reaction medium is restricted by the low solubility of organic compounds. Supercritical and subcritical water is a promising medium to overcome this restriction. When the temperature is elevated and the density is not too high, water mixes well with organic compounds and often acts in a reaction both as an environment and as a reactant. ${ }^{3-8}$ A distinctive feature of supercritical and subcritical water is the availability of a wide range of density and temperature, and the role of water in an organic chemical reaction may be strongly dependent on the thermodynamic state. The reaction control in hydrothermal conditions is then possible only by clarifying the effect of the aqueous medium at the molecular level. Especially, the role of water in simplest organic reactions is the first subject to be investigated in detail toward a systematic construction of hydrothermal organic chemistry.

The simplest organic chemistry is the $\mathrm{C} 1$ chemistry, the chemistry of compounds with single carbon atom. Among the $\mathrm{C} 1$ compounds, formaldehyde and formic acid are of particular interest for their rich abilities in synthetic reactions. Indeed, these two compounds are both aldehyde, and the aldehyde group (with the ketone) constitutes an independent chapter in typical organic chemistry textbooks. ${ }^{9}$ The $\mathrm{C} 1$ conversion schemes involving formaldehyde and formic acid

\footnotetext{
${ }^{a)}$ Author to whom correspondence should be addressed. Electronic mail: nobuyuki@scl.kyoto-u.ac.jp
}

are illustrated in Fig. 1. A characteristic process at hydrothermal conditions is the aldehyde disproportionation reaction expressed as ${ }^{10-13}$

$$
\begin{aligned}
& 2 \mathrm{HCHO}+\mathrm{H}_{2} \mathrm{O} \rightarrow \mathrm{CH}_{3} \mathrm{OH}+\mathrm{HCOOH}, \\
& \mathrm{HCHO}+\mathrm{HCOOH} \rightarrow \mathrm{CH}_{3} \mathrm{OH}+\mathrm{CO}_{2} .
\end{aligned}
$$

In Eq. (1), formaldehyde disproportionates into methanol and formic acid, which are the reduced and oxidized forms of formaldehyde, respectively. The disproportionation between formaldehyde and formic acid takes place through Eq. (2) to produce methanol and carbon dioxide. At ambient conditions, a disproportionation reaction of aldehyde is termed the Cannizzaro reaction and proceeds in the presence of strong base. At hydrothermal conditions, in contrast, we found that aldehyde generally disproportionates into alcohol and acid even under neutral and acidic conditions. ${ }^{10,14,15}$ In the fol-

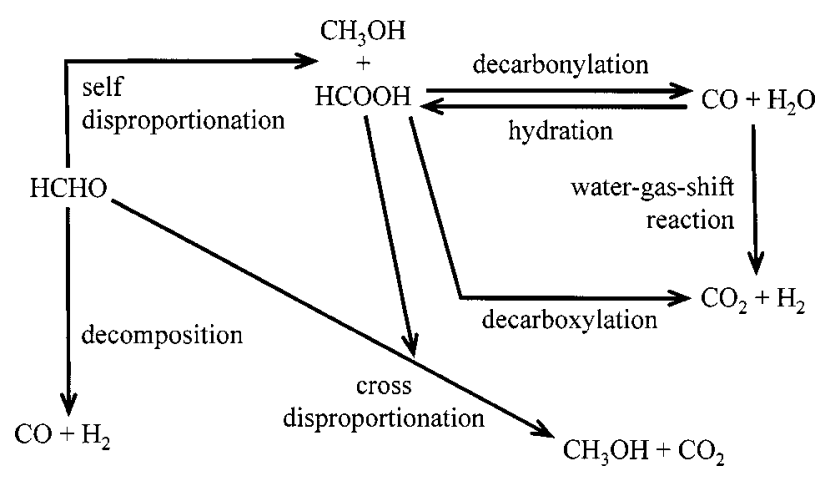

FIG. 1. Illustration of the $\mathrm{C} 1$ conversion processes. 
lowing, we call Eqs. (1) and (2) the self- and crossdisproportionation reactions, respectively, in accordance with the number of reactant aldehyde species involved. Another reactions of importance in Fig. 1 are the decompositions of formaldehyde and formic acid through

$$
\begin{aligned}
& \mathrm{HCHO} \rightarrow \mathrm{CO}+\mathrm{H}_{2}, \\
& \mathrm{HCOOH} \rightarrow \mathrm{CO}+\mathrm{H}_{2} \mathrm{O}, \\
& \mathrm{HCOOH} \rightarrow \mathrm{CO}_{2}+\mathrm{H}_{2} .
\end{aligned}
$$

Equations (4) and (5) represent two competitive pathways for formic acid and generate carbon monoxide and carbon dioxide, respectively. ${ }^{11,16-19}$ In a recent series of papers, we showed that the competition between the decarbonylation (dehydration) and decarboxylation (dehydrogenation) of formic acid depends delicately on the condition of the system and that the decarbonylation is reversible in hydrothermal condition. ${ }^{19,20}$ Formic acid is then a key intermediate of the water-gas-shift reaction ${ }^{20-26}$ given by

$$
\mathrm{CO}+\mathrm{H}_{2} \mathrm{O} \rightarrow \mathrm{CO}_{2}+\mathrm{H}_{2}
$$

and its usage is suggested as a "chemical reservoir" for a clean fuel, $\mathrm{H}_{2}$.

In the present work, we focus on the equilibrium constants of $\mathrm{C} 1$ reactions in water over a wide range of thermodynamic conditions. We evaluate the solvation free energies of the species in Fig. 1, and elucidate the effect of solvent density and temperature on the equilibria of the $\mathrm{C} 1$ conversions. $^{27}$ As shown in Fig. 1, nonpolar and polar compounds are involved in the conversion schemes. In supercritical water, a general tendency was reported for the solvation free energies (excess chemical potentials) of various types of solutes. ${ }^{28}$ The solvation free energy of a nonpolar solute is a steeply increasing function of the solvent density. When the solute is polar, on the other hand, the solvation free energy is not a monotonic function of the density. It decreases in the low-density region, and the dependence is reversed in the high-density region with a minimum in the medium-density region. We construct the equilibrium constant in solution by combining the solvation free energies of the species of interest with their standard free energies of formation in the absence of solvent. A quantitative treatment is then required for the solvation free energy since its difference between the reactant and product sides describes the solvent effect on the equilibrium constant in solution.

When the temperature is high, the reversibility is often attained for a chemical reaction and the thermodynamic condition can be designed to control the yields of desired species. Toward this control, the key information is the equilibrium constant as a function of the thermodynamic condition. The equilibrium constant is notorious, however, for its difficulty of experimental determination, especially at extreme conditions. A theoretical-computational method is then invaluable for the analysis and design of a chemical process if it is accurate and efficient. Once the equilibrium constant is systematically constructed over a wide range of thermodynamic conditions, the reaction control by the solvent will be possible for a reversible process in solution.
In order to explore a wide range of thermodynamic conditions, we calculate the solvation free energy using the method of energy representation developed in previous works. $^{28-31}$ In the method of energy representation, the solvation free energy is provided exactly to second order in solvent density and its accuracy as an approximation is well established for both supercritical and ambient aqueous solutions. Since the approximate method needs the simulations only at the initial and final states of the process of solute insertion, its computational cost is much smaller compared to that in the (formally exact) free energy perturbation or thermodynamic integration method. ${ }^{32}$ Thus, the method of energy representation is suitable for studying the solvation effect over an extensive set of thermodynamic states including supercritical. In the present work, the solvation free energies of the reactive species appearing in Fig. 1 are computationally obtained at hydrothermal conditions. The solvent effect on the equilibrium constants is then determined for the reactions expressed as Eqs. (1)-(6), and the dependence on the (solvent) density and temperature is discussed in connection to the interaction with the solvent water.

\section{THEORETICAL BACKGROUND}

A chemical reaction is a conversion of a set of reactant species into a set of product species. To approach the equilibrium constant, the explicit treatment is necessary only for the reactant and product species. When the overall position and orientation is fixed for the $i$ th reactive species, we let $E_{0 i}$ denote its intramolecular free energy at isolation (in the absence of solvent) and $\Delta \mu_{i}$ describe the solvent effect in the chemical potential. ${ }^{33}$ In the present work, we assume that the electronic structure and molecular geometry of the reactive species (solute) are not coupled with the solvent degrees of freedom. In this approximation, $\Delta \mu_{i}$ is the solvation free energy evaluated under the (effective) solute-solvent interaction, with an appropriate correction to $E_{0 i}$ if needed. ${ }^{27}$ Within the classical statistical mechanics, the solvation free energy $\Delta \mu$ is expressed as

$$
\exp (-\beta \Delta \mu)=\frac{\int d \mathbf{X} \exp \{-\beta[V(\mathbf{X})+U(\mathbf{X})]\}}{\int d \mathbf{X} \exp [-\beta U(\mathbf{X})]},
$$

where $\mathbf{X}$ represents the solvent configuration collectively, the solute-solvent and solvent-solvent interaction energies are $V(\mathbf{X})$ and $U(\mathbf{X})$, respectively, and $\beta$ is the inverse of $k_{B} T$ with the Boltzmann constant $k_{B}$ and the temperature $T$.

When the molarity of the $i$ th reactive species is $\rho_{i}$ and the stoichiometric coefficient is $\nu_{i}$, the condition of chemical equilibrium is written as

$$
\begin{aligned}
& \sum_{i \text { :product }} \nu_{i}\left[E_{0 i}+\Delta \mu_{i}+k_{B} T \ln \left(\rho_{i} / \lambda_{i}\right)\right] \\
& \quad=\sum_{i \text { :reactant }} \nu_{i}\left[E_{0 i}+\Delta \mu_{i}+k_{B} T \ln \left(\rho_{i} / \lambda_{i}\right)\right],
\end{aligned}
$$

where $\lambda_{i}$ is the contribution from the overall translational and rotational energy of the $i$ th species. In the classical approximation for the overall translation and rotation, $\lambda$ is given by 


$$
\lambda=\left\{\begin{array}{l}
\left(\frac{2 \pi M k_{B} T}{h^{2}}\right)^{3 / 2} \frac{8 \pi^{2} I k_{B} T}{\sigma h^{2}} \text { for linear molecule, } \\
\left(\frac{2 \pi M k_{B} T}{h^{2}}\right)^{3 / 2} \frac{\sqrt{\pi}}{\sigma} \prod\left(\frac{8 \pi^{2} I k_{B} T}{h^{2}}\right)^{1 / 2} \text { for nonlinear molecule }
\end{array}\right.
$$

with the symmetry number $\sigma$, the total mass $M$, the moment of inertia $I$ of the molecule, and the Planck constant $h$. The product of Eq. (9) is taken over the rotational degrees of freedom, and I may vary with the rotational degree. The equilibrium constant $K$ in the molarity unit is then expressed as $^{34}$

$$
K=\frac{\prod_{i \text { :product }} \rho_{i}^{\nu_{i}}}{\prod_{i \text { :reactant }} \rho_{i}^{\nu_{i}}}=\frac{\prod_{i \text { :product }} \lambda_{i}^{\nu_{i}}}{\prod_{i \text { :reactant }} \lambda_{i}^{\nu_{i}}} \exp \left[-\beta\left(\Delta E_{0}+\Delta W\right)\right],
$$

where $\Delta E_{0}$ and $\Delta W$ are introduced, respectively, as

$$
\begin{aligned}
& \Delta E_{0}=\sum_{i \text { :product }} \nu_{i} E_{0 i}-\sum_{i \text { :reactant }} \nu_{i} E_{0 i}, \\
& \Delta W=\sum_{i \text { :product }} \nu_{i} \Delta \mu_{i}-\sum_{i \text { :reactant }} \nu_{i} \Delta \mu_{i} .
\end{aligned}
$$

In Eq. (10), the preexponential factor carries the dimension of the equilibrium constant $K$ and depends only on the temperature $T$. Since the intramolecular free energy of the reactive species is assumed to be unaffected by the presence of solvent, the density dependence of $K$ is solely determined by the difference $\Delta W$ in the sum of the solvation free energies between the reactant and product sides. In the present treatment, the reactive species is considered to be either at infinite dilution or coincident with the solvent species. In this case, $K$ depends only on the solvent density and temperature. Even when the solvent molecule appears as a reactive species, it is present in excess in the system and does not violate the validity of Eq. (10) as an expression for the equilibrium constant. $^{35}$

To highlight the solvent effect on the reaction equilibrium, it is convenient to rewrite Eq. (10) as

$$
K=K_{0} \exp (-\beta \Delta W),
$$

where $K_{0}$ is defined as

$$
K_{0}=\frac{\prod_{i: \text { product }} \lambda_{i}^{\nu_{i}}}{\prod_{i \text { reactant }} \lambda_{i}^{\nu_{i}}} \exp \left(-\beta \Delta E_{0}\right)
$$

$K_{0}$ is the equilibrium constant of the reaction in the absence of solvent at the temperature of interest. It can be obtained from a handbook of thermodynamics with an appropriate conversion of the concentration units into molarity.

\section{COMPUTATIONAL PROCEDURES}

The solvent is water. The water molecule was treated as rigid and nonpolarizable, and the SPC/E model was adopted as the intermolecular potential function between water molecules. ${ }^{36}$ The thermodynamic states of interest are specified by the water density and temperature and are listed in Table I. Below the critical temperature, two groups of thermodynamic states were examined. In one of the groups, the temperature is varied at a fixed density of $1.00 \mathrm{~g} / \mathrm{cm}^{3}$, and in the other, the system is on the liquid branch of the (experimental) saturation curve of $\mathrm{H}_{2} \mathrm{O} .{ }^{37}$ Above the critical temperature, the thermodynamic state was explored at a fixed temperature of $400{ }^{\circ} \mathrm{C}$ over a wide density range in Table I.

The solutes are formaldehyde ( $\mathrm{HCHO})$, formic acid $(\mathrm{HCOOH})$, methanol $\left(\mathrm{CH}_{3} \mathrm{OH}\right)$, water $\left(\mathrm{H}_{2} \mathrm{O}\right)$, hydrogen $\left(\mathrm{H}_{2}\right)$, carbon monoxide $(\mathrm{CO})$, and carbon dioxide $\left(\mathrm{CO}_{2}\right)$. They appear as the reactive species in Fig. 1 and Eqs. (1)-(6). The intermolecular interaction was assumed to be pairwise additive and was described with Coulombic and Lennard-Jones terms. The molecular geometry and the potential parameters (partial charges and Lennard-Jones $\epsilon$ and $\sigma$ ) for HCHO were set to those given in Ref. 38. When the solute is $\mathrm{HCOOH}$, the cis geometry in the notation of Ref. 39 was adopted (in which the carbonyl $\mathrm{O}$ and the hydroxy $\mathrm{H}$ are on the same side) and the revised potential parameter set in Ref. 40 was employed. At the transition state of the formic acid decomposition, the structure of the formic acid-water complex was a focus of previous works. ${ }^{41-43}$ In the present work, $\mathrm{HCOOH}$ is either an initial or final species in the reaction and its most stable geometry was explicitly treated. The parameters for $\mathrm{CH}_{3} \mathrm{OH}$ and $\mathrm{H}_{2}$ were taken from Refs. 44 and 45 , respectively. Note that a single-site Lennard-Jones interaction was used for the $\mathrm{H}_{2}$ molecule. $\mathrm{CO}$ and $\mathrm{CO}_{2}$ were described as two- and three-site molecules, respectively, and the interaction sites were placed at the $\mathrm{C}$ and $\mathrm{O}$ positions. The $\mathrm{C}-\mathrm{O}$ bond length was fixed at the gas-phase experimental value of $1.128 \AA$ for $\mathrm{CO}$ and $1.160 \AA$ for $\mathrm{CO}_{2}{ }^{46}$ The Lennard-Jones $\epsilon$ and $\sigma$ for the $\mathrm{C}$ and $\mathrm{O}$ sites were set at the EPM values in Table I of Ref. 47, and the charge on the oxygen site was 0.02 for $\mathrm{CO}$ and -0.32 for $\mathrm{CO}_{2}$ in the unit of elementary charge. These charges were determined to reproduce the gas-phase experimental dipole ${ }^{46}$ and quadrupole ${ }^{48}$ moments, respectively, and the charge neutrality led to the value of the $\mathrm{C}$-site charge. The Lennard-Jones part of the solute-water potential function was then constructed by the standard Lorentz-Berthelot combining rule. ${ }^{49}$

For each solute and at each thermodynamic state, Monte Carlo simulations of the solution and pure solvent systems were conducted in the canonical ensemble. In the simulation of the solution system, the standard Metropolis sampling scheme was implemented by locating one solute molecule of interest and 500 water molecules in a cubic unit cell. ${ }^{32}$ The simulation length was $500 \mathrm{~K}$ passes, where one pass corre- 
TABLE I. Solvation free energy in the dimensionless form of $\beta \Delta \mu$. Each value is rounded to a multiple of 0.1 . The error is estimated at $95 \%$ confidence level, and is not shown when its rounded value is zero.

\begin{tabular}{|c|c|c|c|c|c|c|c|c|}
\hline \multicolumn{2}{|c|}{ Thermodynamic state } & \multicolumn{7}{|c|}{ Solute } \\
\hline Density $\left(\mathrm{g} / \mathrm{cm}^{3}\right)$ & Temperature $\left({ }^{\circ} \mathrm{C}\right)$ & $\mathrm{CH}_{3} \mathrm{OH}$ & $\mathrm{HCHO}$ & $\mathrm{HCOOH}$ & $\mathrm{H}_{2} \mathrm{O}$ & $\mathrm{CO}$ & $\mathrm{CO}_{2}$ & $\mathrm{H}_{2}$ \\
\hline \multicolumn{9}{|c|}{ Temperature variation at a fixed density } \\
\hline 1.00 & 25 & $-8.0 \pm 0.2$ & $-3.3 \pm 0.1$ & $-8.5 \pm 0.3$ & $-13.3 \pm 0.4$ & $3.6 \pm 0.1$ & $1.6 \pm 0.1$ & 4.3 \\
\hline 1.00 & 100 & $-3.6 \pm 0.2$ & $-0.9 \pm 0.1$ & $-4.6 \pm 0.1$ & $-8.7 \pm 0.1$ & 4.4 & 3.2 & 4.5 \\
\hline 1.00 & 150 & $-1.7 \pm 0.1$ & $0.3 \pm 0.1$ & $-2.5 \pm 0.1$ & $-6.6 \pm 0.2$ & 4.7 & $3.9 \pm 0.1$ & 4.6 \\
\hline 1.00 & 200 & $-0.2 \pm 0.1$ & $1.3 \pm 0.1$ & $-1.0 \pm 0.1$ & $-5.0 \pm 0.1$ & 5.0 & 4.5 & 4.6 \\
\hline 1.00 & 250 & $0.9 \pm 0.1$ & 2.1 & $0.1 \pm 0.1$ & -3.6 & 5.1 & 5.0 & 4.6 \\
\hline 1.00 & 300 & 1.8 & 2.7 & $1.2 \pm 0.1$ & -2.6 & 5.3 & 5.4 & 4.6 \\
\hline 1.00 & 350 & 2.5 & $3.3 \pm 0.1$ & $1.9 \pm 0.1$ & -1.7 & 5.4 & 5.7 & 4.6 \\
\hline \multicolumn{9}{|c|}{ State change along the liquid branch of the saturation curve } \\
\hline 0.96 & 100 & $-4.4 \pm 0.1$ & $-1.4 \pm 0.1$ & -5.1 & $-9.0 \pm 0.2$ & 3.9 & $2.6 \pm 0.1$ & 4.1 \\
\hline 0.92 & 150 & $-2.9 \pm 0.1$ & $-0.9 \pm 0.1$ & $-3.6 \pm 0.1$ & $-7.1 \pm 0.1$ & 3.7 & 2.7 & 3.7 \\
\hline 0.86 & 200 & $-2.0 \pm 0.1$ & $-0.6 \pm 0.1$ & -2.9 & $-5.6 \pm 0.1$ & 3.3 & 2.5 & 3.2 \\
\hline 0.80 & 250 & -1.5 & $-0.3 \pm 0.1$ & $-2.2 \pm 0.1$ & $-4.6 \pm 0.1$ & 2.9 & 2.2 & 2.8 \\
\hline 0.71 & 300 & $-1.4 \pm 0.1$ & -0.4 & $-1.9 \pm 0.1$ & -3.8 & 2.3 & 1.8 & 2.2 \\
\hline 0.57 & 350 & -1.3 & -0.6 & -1.7 & -3.1 & 1.5 & 1.1 & 1.5 \\
\hline \multicolumn{9}{|c|}{ Density variation at a fixed temperature } \\
\hline 1.20 & 400 & 6.6 & 7.0 & 5.9 & 0.7 & 8.2 & 9.5 & 6.8 \\
\hline 1.00 & 400 & $3.1 \pm 0.1$ & 3.7 & 2.6 & -1.0 & 5.4 & 5.9 & 4.6 \\
\hline 0.80 & 400 & 0.7 & 1.3 & $0.2 \pm 0.1$ & -2.2 & 3.3 & 3.2 & 2.9 \\
\hline 0.60 & 400 & -0.7 & -0.1 & -1.1 & $-2.5 \pm 0.1$ & 1.8 & 1.5 & 1.7 \\
\hline 0.40 & 400 & -1.1 & -0.7 & $-1.4 \pm 0.1$ & -2.3 & 0.9 & 0.6 & 0.9 \\
\hline 0.20 & 400 & -1.0 & -0.7 & -1.1 & -1.7 & 0.4 & 0.2 & 0.4 \\
\hline 0.10 & 400 & -0.6 & -0.5 & -0.8 & -1.1 & 0.2 & 0.0 & 0.2 \\
\hline
\end{tabular}

sponds to the generation of 500 configurations, and the method of preferential sampling was not adopted. The periodic boundary condition was employed in the minimum image convention, and the electrostatic potential was handled by the Ewald method with the surrounding medium of infinite dielectric constant. The screening parameter was then set to $5 / L$, where $L$ is the length of the unit cell, and 514 reciprocal lattice vectors were used. The truncation at $L / 2$ was applied on the site-site basis to the real-space part of the electrostatic interaction in the Ewald method and the Lennard-Jones part of the intermolecular interaction. To simulate the pure solvent system, 500 water molecules were located in a cubic unit cell and were sampled for $100 \mathrm{~K}$ passes with the Metropolis scheme. The size of the unit cell was identical to that of the corresponding simulation of the solution system consisting of one solute molecule and 500 water molecules, and the boundary condition and Ewald sum parameters were the same as those for the solution system. The solvation free energy was then approximately evaluated in the method of energy representation according to the procedure presented in previous papers. ${ }^{28,30}$

\section{RESULTS AND DISCUSSION}

\section{A. Solvation free energy}

In this section, we first observe the density and temperature dependence of the solvation free energy $\Delta \mu$. Table I lists $\beta \Delta \mu$ calculated in the present work, where $\beta$ is the inverse of the thermal energy $k_{B} T$. The dimensionless description is provided because the solvation free energy appears in the form of $\beta \Delta \mu$ in the expression for the equilibrium constant given by Eq. (10). The overall trend of the density and temperature dependence of $\beta \Delta \mu$ agrees with that reported in Ref. 28. At constant temperature, $\beta \Delta \mu$ of a nonpolar solute is a strongly increasing function of the solvent density, and the density dependence is not monotonic for a polar solute. At a fixed solvent density, on the other hand, $\beta \Delta \mu$ of a polar solute increases rapidly with the temperature elevation, while the temperature dependence is weaker for a nonpolar solute. $^{50}$

The density dependence of $\beta \Delta \mu$ at a fixed supercritical temperature of $400{ }^{\circ} \mathrm{C}$ is illustrated in Fig. 2 for typical cases of $\mathrm{HCOOH}, \mathrm{H}_{2} \mathrm{O}, \mathrm{CO}$, and $\mathrm{CO}_{2}$. It is seen in Table I and Fig. 2 that the density dependence at constant temperature is qualitatively different between polar and nonpolar species. ${ }^{51}$ When the solute is nonpolar, $\beta \Delta \mu$ increases steeply with the density in the range examined. In contrast, $\beta \Delta \mu$ of a polar solute is not a monotonic function of the density. It decreases in the low-density region and involves a minimum in the medium-density region. The density corresponding to the minimum is larger when the solute-water interaction is more favorable. ${ }^{52}$ In the high-density region, $\beta \Delta \mu$ of a polar solute increases with the density, and the density dependence of $\beta \Delta \mu$ is qualitatively common to the polar and nonpolar solutes. In the Appendix, the density dependence of $\Delta \mu$ is further discussed in connection to the solute-solvent radial distribution function.

The temperature dependence of $\beta \Delta \mu$ below the critical 


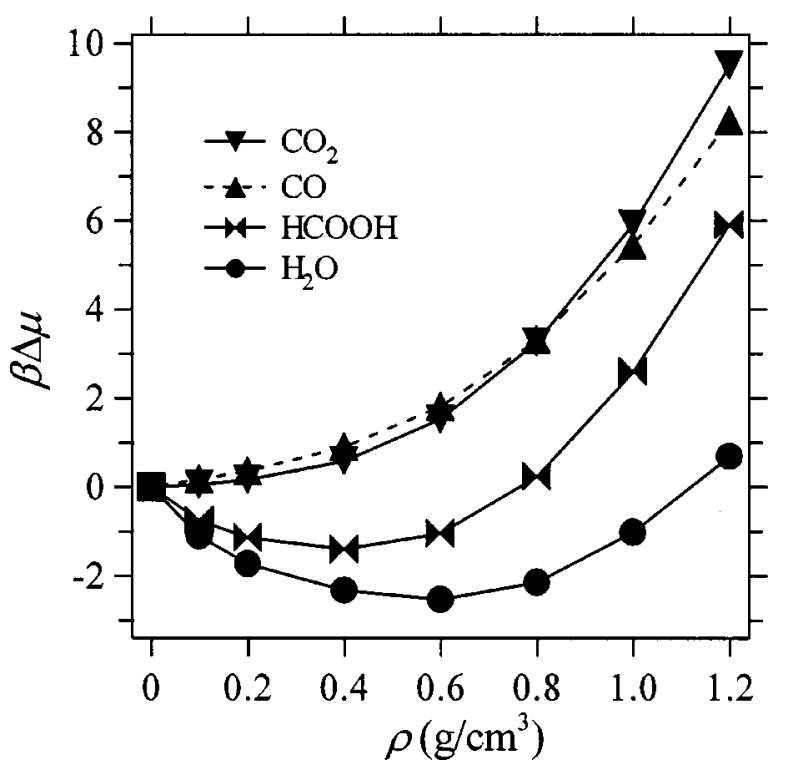

FIG. 2. $\beta \Delta \mu$ for $\mathrm{HCOOH}, \mathrm{H}_{2} \mathrm{O}, \mathrm{CO}$, and $\mathrm{CO}_{2}$ as a function of the solvent density $\rho$ at a fixed temperature of $400{ }^{\circ} \mathrm{C}$. The error bar at $95 \%$ confidence level is smaller than the size of the corresponding data symbol. The lines connecting the data are drawn for the eye guide. $\Delta \mu=0$ holds at $\rho=0$ by definition.

is illustrated in Fig. 3 for typical cases of $\mathrm{CH}_{3} \mathrm{OH}, \mathrm{CO}$, and $\mathrm{CO}_{2}$. According to Table I and Fig. 3, $\beta \Delta \mu$ of each solute examined becomes less favorable (more positive) with the temperature elevation at a fixed density of $1.00 \mathrm{~g} / \mathrm{cm}^{3}$. The temperature dependence is weak for $\mathrm{CO}$ and $\mathrm{H}_{2}$ and is strong for the polar solutes. To interpret this point, it is to be noted that $\beta \Delta \mu$ involves two competitive contributions from the repulsive and attractive interactions between the solute and solvent. The repulsive interaction is typically described as the excluded volume effect, and its strength in the dimensionless description is rather insensitive to the temperature

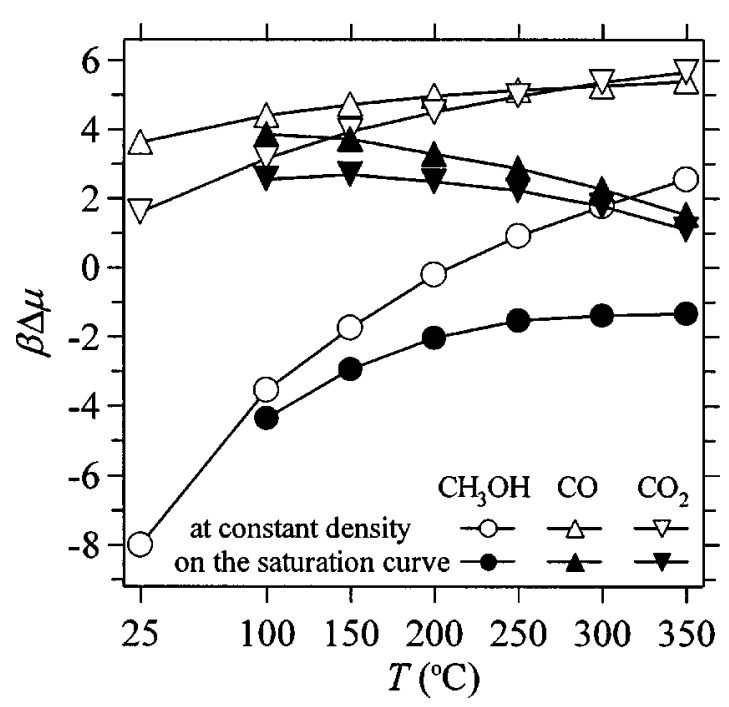

FIG. 3. $\beta \Delta \mu$ for $\mathrm{CH}_{3} \mathrm{OH}, \mathrm{CO}$, and $\mathrm{CO}_{2}$ as a function of the temperature $T$ below the critical. The filled symbols represent the states with a fixed solvent density of $1.00 \mathrm{~g} / \mathrm{cm}^{3}$, and the open symbols represent the states on the liquid branch of the (experimental) saturation curve of $\mathrm{H}_{2} \mathrm{O}$. The error bar at $95 \%$ confidence level is smaller than the size of the corresponding data symbol. The lines connecting the data are drawn for the eye guide. variation. The attractive interaction for the solute molecules in Table I, on the other hand, competes against the thermal energy and responds more sensitively to the temperature change. When the solute is nonpolar, ${ }^{50}$ the repulsive interaction makes the major contribution to $\beta \Delta \mu$ and leads to the weak temperature dependence. The attractive interaction is more effectively operative for a polar solute and is responsible for the stronger dependence of $\beta \Delta \mu$ on the temperature. It is of particular interest to see that $\beta \Delta \mu$ of $\mathrm{CO}_{2}$ increases rapidly in the temperature elevation from 25 to $\sim 150{ }^{\circ} \mathrm{C}$ and exhibits a weaker dependence at higher temperatures. As illustrated by using radial distribution functions in a previous work, ${ }^{53}$ the partial charges of $\mathrm{CO}_{2}$ are large and the attractive interaction between $\mathrm{CO}_{2}$ and water is stronger than that for typical nonpolar solutes such as $\mathrm{CO}$ and $\mathrm{H}_{2}$. In the low-temperature region, the relatively sensitive dependence of $\beta \Delta \mu$ on the temperature is ascribed to the presence of the $\mathrm{CO}_{2}$-water attraction. In the hightemperature region, in contrast, the repulsive interaction is more effective and the temperature dependence of $\beta \Delta \mu$ becomes close to that for other nonpolar solutes.

At the temperatures below the critical in Table I, $\beta \Delta \mu$ of each solute is more favorable (more negative) on the liquid branch of the saturation curve than at a fixed density of $1.00 \mathrm{~g} / \mathrm{cm}^{3}$. The saturation curve in the temperature range of Table I is then considered to be located in the high-density and high-temperature region in the sense that $\beta \Delta \mu$ increases with the density at constant temperature. When the temperature dependence of $\beta \Delta \mu$ is examined for the nonpolar solutes on the saturation curve, the results in Table I and Fig. 3 are in agreement with previous experimental and computational observations. ${ }^{54-58}$ The agreement includes, in particular, the inversions of $\beta \Delta \mu$ and the corresponding Henry's constant for $\mathrm{CO}$ and $\mathrm{CO}_{2}$ at $100-200{ }^{\circ} \mathrm{C} .{ }^{59,60}$ This illustrates the reliability of the present methodology. Above $\sim 200{ }^{\circ} \mathrm{C}$, the temperature elevation along the saturation curve leads to a less favorable $\beta \Delta \mu$ for a polar solute and to a more favorable $\beta \Delta \mu$ for a nonpolar solute. An exception is seen for HCHO above $\sim 300^{\circ} \mathrm{C}$ and reflects the nonmonotonic density dependence of $\beta \Delta \mu$ at constant temperature. Actually, the opposite temperature dependence between the polar and nonpolar solutes is in contrast to the subcritical results in Table I and Figs. 2 and 3 that the temperature effect at constant density and the density effect at constant temperature are qualitatively similar between the polar and nonpolar solutes. When the temperature dependence along the saturation curve is viewed as a combination of the temperature effect at constant density and the density effect at constant temperature, the quantitative difference in the strengths of the two effects is responsible for the qualitative difference in the temperature dependence of $\beta \Delta \mu$ along the saturation curve. The temperature effect overwhelms the density effect when the solute is polar, while the contrary is operative for a nonpolar solute.

\section{B. Solvent effect on the reaction equilibria}

In this section, we describe the solvent effect on the reaction equilibria of Eqs. (1)-(6). The description is pro- 

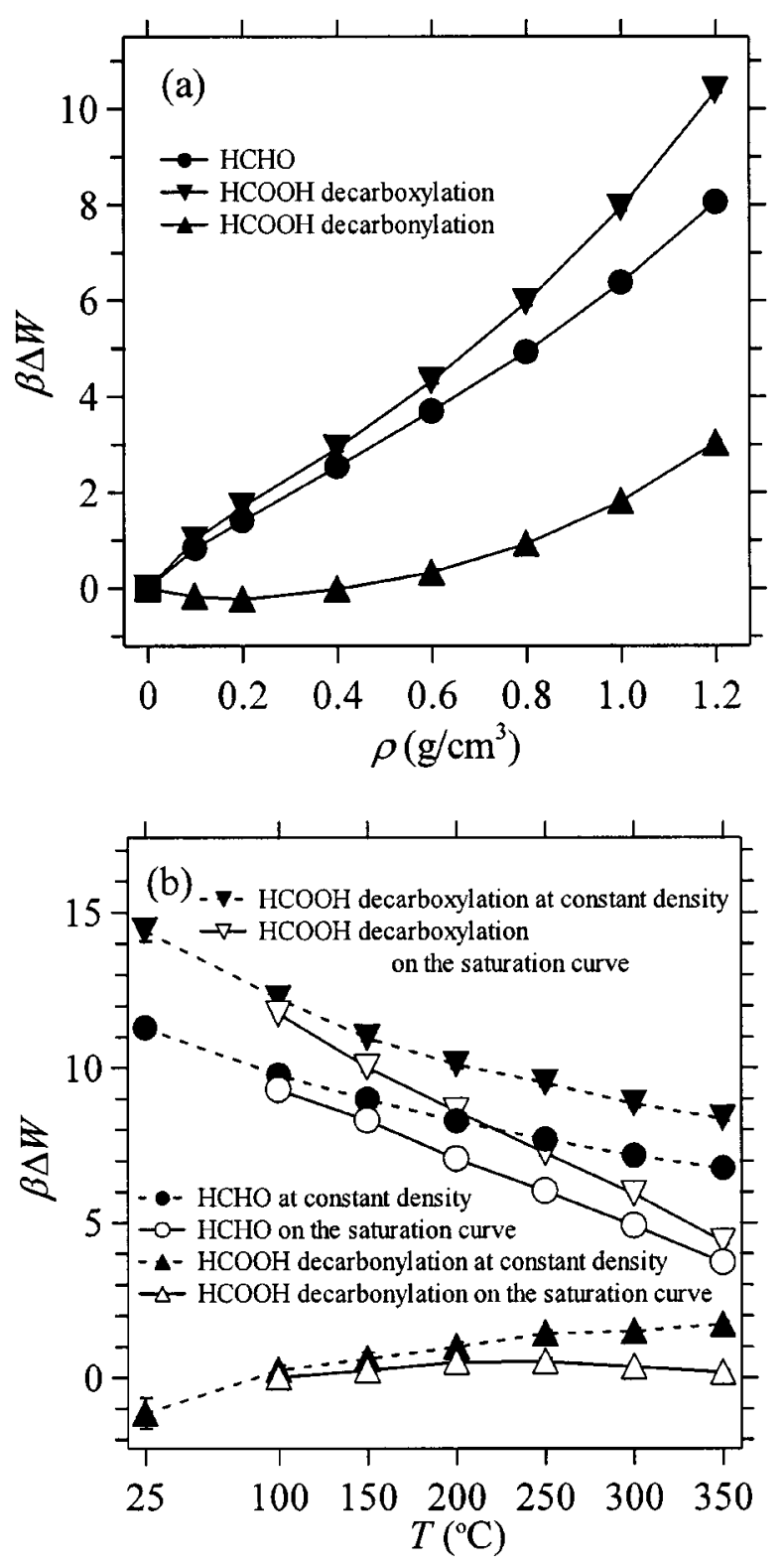

FIG. 4. The solvent effect $\beta \Delta W$ for the decompositions of formaldehyde and formic acid (a) as a function of the solvent density $\rho$ and (b) as a function of the temperature $T$ below the critical. In (a), the temperature is fixed at $400{ }^{\circ} \mathrm{C}$. In (b), the filled symbols represent the states with a fixed solvent density of $1.00 \mathrm{~g} / \mathrm{cm}^{3}$, and the open symbols represent the states on the liquid branch of the (experimental) saturation curve of $\mathrm{H}_{2} \mathrm{O}$. The error bar is expressed at $95 \%$ confidence level, and is smaller than the size of the corresponding data symbol when it is not shown. The lines connecting the data are drawn for the eye guide. $\Delta W=0$ holds at $\rho=0$ by the definition of $\Delta \mu$.

vided in terms of a free-energy change $\Delta W$ introduced by Eq. (12). We present the dependence of $\beta \Delta W$ on the (solvent) density and temperature. The dimensionless presentation is adopted since the equilibrium constant given by Eq. (10) involves the solvent effect in the form of $\beta \Delta W$.

In Fig. 4, we show $\beta \Delta W$ for the decomposition processes of formaldehyde and formic acid expressed as Eqs. (3)-(5). The HCHO decomposition (decarbonylation) and the $\mathrm{HCOOH}$ decarboxylation given by Eqs. (3) and (5), respectively, correspond to the conversion of one polar species into two nonpolar. These two processes respond similarly to the change in the thermodynamic state, and their $\beta \Delta W$ vary by $\sim 10$ with the density and temperature in Fig. 4 . The solvent water serves to suppress the production of nonpolar species, and its effect is stronger at a higher density and/or a lower temperature. The $\mathrm{HCOOH}$ decarbonylation is, on the other hand, a decomposition of one polar species into one polar and one nonpolar, as shown by Eq. (4). The dependence of $\beta \Delta W$ on the thermodynamic state is then relatively weak due to the cancellation of the $\beta \Delta \mu$ changes in the reactant and product sides. Actually, $\beta \Delta W$ for the $\mathrm{HCOOH}$ decarbonylation reflects the delicate balance between the reactant and product $\beta \Delta \mu$, and is not necessarily monotonic in Fig. 4. When the reaction equilibrium is concerned in the absence of solvent, ${ }^{34}$ the corresponding standard free-energy change is estimated to be $\sim-10$ for Eqs. (3) and (5) and $\sim-5$ for Eq. (4) in the unit of the thermal energy $k_{B} T$. $^{61,62}$ These values are comparable to $\beta \Delta W$ in Fig. 4. The figure thus provides a possibility that the reaction equilibria for the $\mathrm{HCHO}$ and $\mathrm{HCOOH}$ decompositions can be controlled by tuning the (solvent) density and temperature.

In the two decomposition pathways of $\mathrm{HCOOH}$ expressed as Eqs. (4) and (5), the conversion between their product sides is called the water-gas-shift reaction and is important both in fundamental physical chemistry and in fuel technology. ${ }^{20-26}$ The water-gas-shift reaction given by Eq. (6) involves $\mathrm{HCOOH}$ as an intermediate, ${ }^{20}$ and its equilibrium constant determines the relative weights of the equilibrium decompositions of $\mathrm{HCOOH}$ into $\mathrm{CO}$ and $\mathrm{CO}_{2}$. In Fig. 5 , we show $\beta \Delta W$ for the water-gas-shift reaction. The solvent favors the reactant side of Eq. (6) over the whole thermodynamic range examined, and acts more strongly at a higher density and/or a lower temperature. $\Delta W$ can actually be controlled on the order of $10 \mathrm{kcal} / \mathrm{mol}$ through the change in the (solvent) density and temperature. When no solvent is employed, the standard free-energy change of Eq. (6) is estimated to be $\sim-5 \mathrm{kcal} / \mathrm{mol}$, in favor of the $\mathrm{CO}_{2}$ side. ${ }^{61,62}$ This value is comparable in magnitude to $\Delta W$ in Fig. 5 , and the favorable side of Eq. (6) can even be overturned by the solvent water. The strong solvent effect in the water-gas-shift reaction may be understood by noting that the reaction is a transformation of one polar and one nonpolar species into two nonpolar. As seen in Table I, the solvation free energy $\Delta \mu$ is relatively similar among the nonpolar solutes, $\mathrm{CO}$, $\mathrm{CO}_{2}$, and $\mathrm{H}_{2}$, at high temperatures. $\Delta W$ of the hydrothermal water-gas-shift reaction is then essentially the difference of $\Delta \mu$ of one nonpolar species from that of polar $\mathrm{H}_{2} \mathrm{O}$. Since the sign is opposite between the $\Delta \mu$ of the nonpolar solute and $\mathrm{H}_{2} \mathrm{O}$, the two $\Delta \mu$ effects enhance each other. When the density is increased at a fixed temperature of $400{ }^{\circ} \mathrm{C}$, Fig. 5 shows that the equilibrium shifts to the reactant side. This is in agreement with the experimental results of Hirth and Franck performed at $500-600{ }^{\circ} \mathrm{C},{ }^{24,63}$ and illustrates the significance of the solvation effect even at high-temperature conditions.

The characteristic reaction for the $\mathrm{C} 1$ aldehyde is disproportionation. HCHO exhibits the self-disproportionation given by Eq. (1), and the cross-disproportionation expressed as Eq. (2) takes place for $\mathrm{HCHO}$ and $\mathrm{HCOOH}$. In Fig. 6, we show $\beta \Delta W$ for the two disproportionation reactions. The self-disproportionation is more favored by solvent than the 

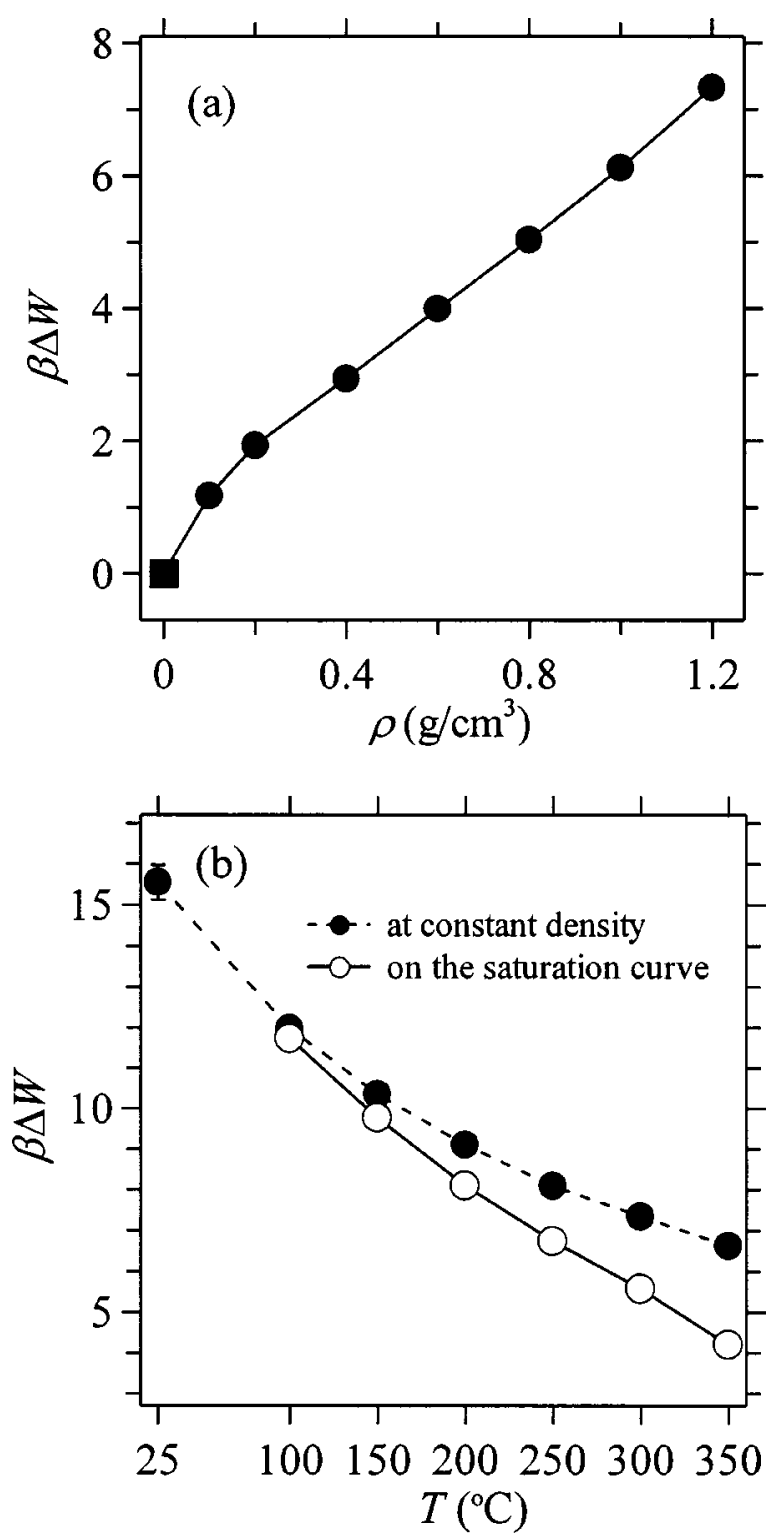

FIG. 5. The solvent effect $\beta \Delta W$ for the water-gas-shift reaction (a) as a function of the solvent density $\rho$ and (b) as a function of the temperature $T$ below the critical. In (a), the temperature is fixed at $400{ }^{\circ} \mathrm{C}$. In (b), the filled symbols represent the states with a fixed solvent density of $1.00 \mathrm{~g} / \mathrm{cm}^{3}$, and the open symbols represent the states on the liquid branch of the (experimental) saturation curve of $\mathrm{H}_{2} \mathrm{O}$. The error bar is expressed at $95 \%$ confidence level, and is smaller than the size of the corresponding data symbol when it is not shown. The lines connecting the data are drawn for the eye guide. $\Delta W=0$ holds at $\rho=0$ by the definition of $\Delta \mu$.

cross, and $\Delta W$ varies on the order of a few $k_{B} T$. The solvent suppresses the disproportionation reactions with positive $\Delta W$, except in the high-density and high-temperature region for the self. Actually, the self-disproportionation is a conversion of three polar species into two polar. $\Delta W$ then reduces with the density elevation beyond $\sim 0.4 \mathrm{~g} / \mathrm{cm}^{3}$ at high temperatures since the solvation free energy of a polar species becomes unfavorable with the density in that thermodynamic region.

\section{Equilibrium constant}

In this section, we present the equilibrium constants for the $\mathrm{C} 1$ reactions expressed as Eqs. (1)-(6). In the absence of
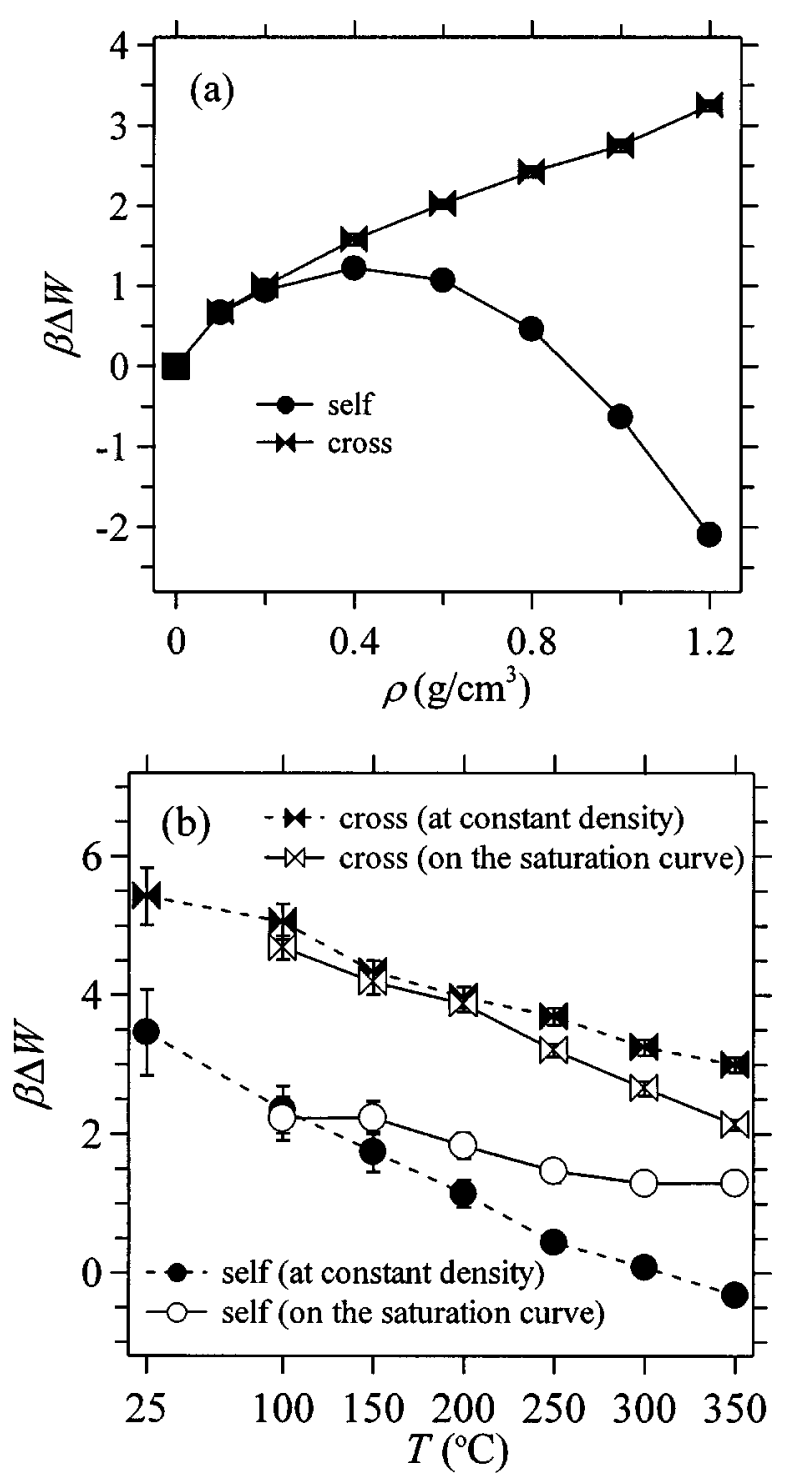

FIG. 6. The solvent effect $\beta \Delta W$ for the disproportionation reactions (a) as a function of the solvent density $\rho$ and (b) as a function of the temperature $T$ below the critical. In (a), the temperature is fixed at $400{ }^{\circ} \mathrm{C}$. In (b), the filled symbols represent the states with a fixed solvent density of $1.00 \mathrm{~g} / \mathrm{cm}^{3}$, and the open symbols represent the states on the liquid branch of the (experimental) saturation curve of $\mathrm{H}_{2} \mathrm{O}$. The error bar is expressed at $95 \%$ confidence level, and is smaller than the size of the corresponding data symbol when it is not shown. The lines connecting the data are drawn for the eye guide. $\Delta W=0$ holds at $\rho=0$ by the definition of $\Delta \mu$.

solvent, the equilibrium constant $K_{0}$ is given by Eq. (14) and is constructed from the standard free energy of formation for each species of interest in the dilute gas condition. ${ }^{61,62} K_{0}$ is a function only of the temperature, and is combined through Eq. (13) with $\Delta W$ treated in the previous section to provide the equilibrium constant $K$ in the presence of solvent. It should be noted that $K$ and $K_{0}$ are in general not dimensionless and need to be treated with explicit specification of the unit. In the following, we adopt the molarity unit for $K$ and $K_{0}$ and express them numerically in (a power of) $\mathrm{mol} / \mathrm{L}$. We will show $\ln K$ and $\ln K_{0}$ as functions of the temperature between 25 and $400{ }^{\circ} \mathrm{C}$. The solvent density is then 1.00 and $0.40 \mathrm{~g} / \mathrm{cm}^{3}$ at 25 and $400{ }^{\circ} \mathrm{C}$, respectively, and is set to the one on the liquid branch of the (experimental) saturation curve of $\mathrm{H}_{2} \mathrm{O}$ at the other temperatures. The density depen- 


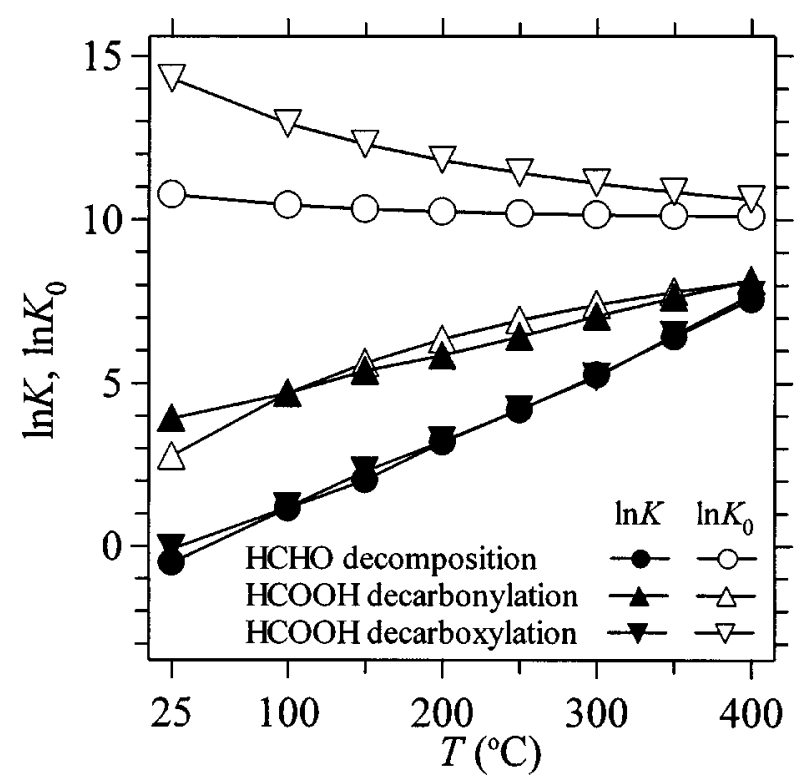

FIG. 7. The equilibrium constants $\ln K$ and $\ln K_{0}$ as functions of the temperature $T$ for the decompositions of formaldehyde and formic acid. The solvent density is 1.00 and $0.40 \mathrm{~g} / \mathrm{cm}^{3}$ at 25 and $400{ }^{\circ} \mathrm{C}$, respectively, and is set to the one on the liquid branch of the (experimental) saturation curve of $\mathrm{H}_{2} \mathrm{O}$ at the other temperatures. The lines connecting the data are drawn for the eye guide.

dence of $\ln K$ will not be shown explicitly since it is solely carried by $\beta \Delta W$ and is parallel to the density dependence of $-\beta \Delta W$ with an offset of $\ln K_{0}$.

In Fig. 7, we show $\ln K$ and $\ln K_{0}$ for the decomposition processes of $\mathrm{HCHO}$ and $\mathrm{HCOOH}$ described by Eqs. (3)-(5). The solvent is in favor of the reactant sides of the $\mathrm{HCHO}$ decomposition (decarbonylation) and the $\mathrm{HCOOH}$ decarboxylation given by Eqs. (3) and (5), respectively. The solvent effect shown in Fig. 7 amounts to 5-10 in the $\ln K$ unit for these two processes, and the temperature dependence of $\ln K$ is reversed from that of $\ln K_{0}$. The difference between $\ln K$ and $\ln K_{0}$ is relatively small, on the other hand, for the $\mathrm{HCOOH}$ decarbonylation expressed as Eq. (4). Actually, the solvent can change the preference order of the $\mathrm{HCOOH}$ decarbonylation and decarboxylation at equilibrium. In Fig. 7, $\ln K$ is larger for the decarbonylation than for the decarboxylation, while the opposite holds for $\ln K_{0}$. When the temperature is below $\sim 300{ }^{\circ} \mathrm{C}$, the $\ln K$ values for the $\mathrm{HCOOH}$ decompositions are less than $\sim 5$ in Fig. 7 . The detection of $\mathrm{HCOOH}$ is then expected to be feasible in its hydrothermal decomposition mixture. Indeed, we were experimentally successful in detecting $\mathrm{HCOOH}$ and demonstrating its role as an intermediate of the water-gas-shift reaction. ${ }^{20,64}$

In the $\mathrm{HCOOH}$ decompositions expressed as Eqs. (4) and (5), the conversion between the product sides is the water-gas-shift reaction given by Eq. (6). Its equilibrium constant determines the relative weights at equilibrium of the competitive decompositions of $\mathrm{HCOOH}$ into $\mathrm{CO}$ and $\mathrm{CO}_{2}$. In Fig. 8, we show $\ln K$ and $\ln K_{0}$ for the water-gas-shift reaction. The solvent effect amounts to 5-15 at the thermodynamic states shown. Actually, the solvent can overturn the favorable side of the equilibrium in our convention of $K$ and $K_{0}$. It is seen in Figs. 5 and 8 that although the $\mathrm{CO}_{2}$ side is more stable in the absence of solvent, the $\mathrm{CO}$ side becomes

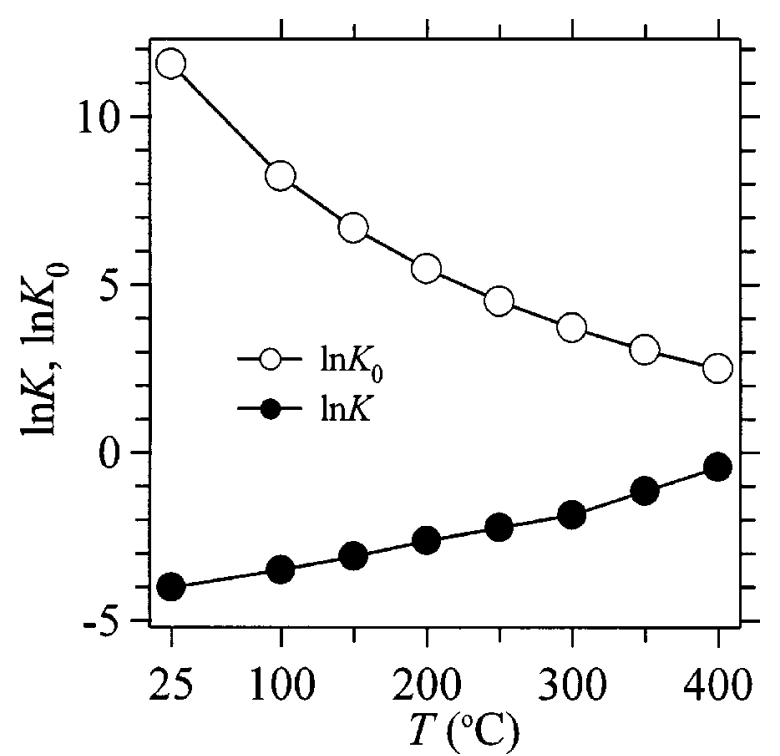

FIG. 8. The equilibrium constants $\ln K$ and $\ln K_{0}$ as functions of the temperature $T$ for the water-gas-shift reaction. The solvent density is 1.00 and $0.40 \mathrm{~g} / \mathrm{cm}^{3}$ at 25 and $400{ }^{\circ} \mathrm{C}$, respectively, and is set to the one on the liquid branch of the (experimental) saturation curve of $\mathrm{H}_{2} \mathrm{O}$ at the other temperatures. The lines connecting the data are drawn for the eye guide.

more favorable with the solvent, except in the low-density supercritical region below $\sim 0.3 \mathrm{~g} / \mathrm{cm}^{3}$. When the density is low enough and/or the temperature is high enough, $K$ approaches to $K_{0}$. Figures 5 and 8 thus show that the equilibrium of the water-gas-shift reaction can be tuned by the (solvent) density and temperature.

A strong solvent effect on the water-gas-shift reaction is also evidenced by the temperature dependence of the equilibrium constant. In Fig. 8, the temperature elevation leads to an increase of $\ln K$ and a decrease of $\ln K_{0}$. The solvent effect is large enough to reverse the dependence of the equilibrium constant on the temperature. Actually, the temperature dependence of $\ln K$ in Fig. 8 is in agreement with the experimental observation at hydrothermal conditions that the $\mathrm{CO}_{2}$ side is more favorable at higher temperatures. ${ }^{11,16-20}$ The explicit treatment of the solvation effect is thus necessary to assess the temperature effect on the equilibrium of the water-gas-shift reaction.

Finally, Fig. 9 presents $\ln K$ and $\ln K_{0}$ for the two disproportionation reactions given by Eqs. (1) and (2). The solvent effect is relatively weak, and the deviation of $\ln K$ from $\ln K_{0}$ is small in the overall trend. In this case, the reaction equilibria are essentially determined electronically and the solvent is needed only for the activation.

\section{CONCLUSIONS}

The reaction equilibria of the hydrothermal chemistry concerning formaldehyde and formic acid have been analyzed computationally with emphasis on the solvent effect. It is found that the solvent suppresses the HCHO decomposition and the $\mathrm{HCOOH}$ decarboxylation processes, while the solvent effect is relatively weak for the $\mathrm{HCOOH}$ decarbonylation. This is caused by the characteristic difference in the solvation free energies of the species involved, and the production of nonpolar species is inhibited by the solvent water. 


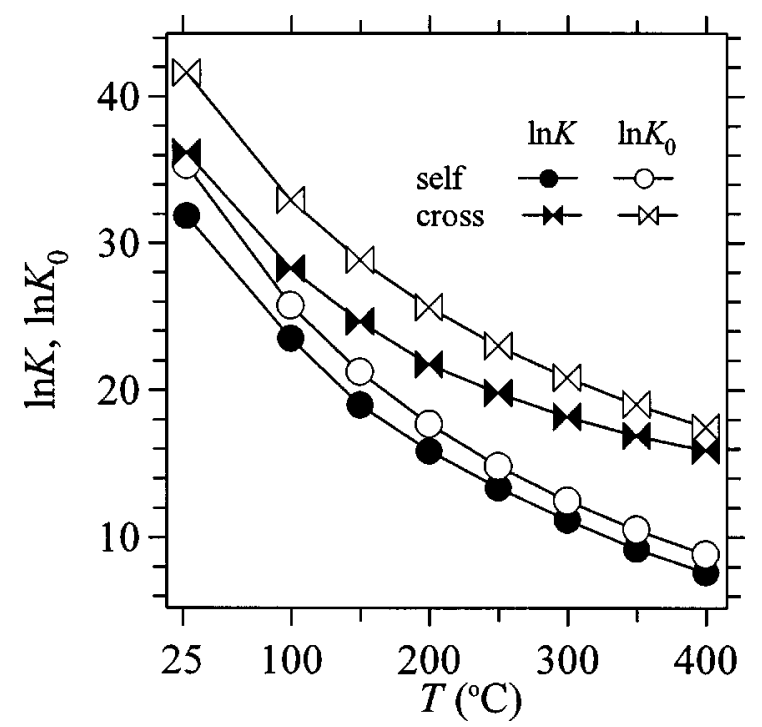

FIG. 9. The equilibrium constants $\ln K$ and $\ln K_{0}$ as functions of the temperature $T$ for the disproportionation reactions. The solvent density is 1.00 and $0.40 \mathrm{~g} / \mathrm{cm}^{3}$ at 25 and $400{ }^{\circ} \mathrm{C}$, respectively, and is set to the one on the liquid branch of the (experimental) saturation curve of $\mathrm{H}_{2} \mathrm{O}$ at the other temperatures. The lines connecting the data are drawn for the eye guide.

Actually, the solvent controls the equilibrium of the watergas-shift reaction. The equilibrium constants in the absence and presence of solvent show that the more stable side of the reaction can be tuned by the solvent density and temperature. The reaction equilibrium is dominated by the electronic part, in contrast, for the aldehyde disproportionations.

Among the compounds appearing in Fig. 1, carbon monoxide and hydrogen are the simplest. Their mixture is called the synthesis gas, and is the source of the methane and methanol generations expressed, respectively, as ${ }^{21}$

$$
\begin{aligned}
& \mathrm{CO}+3 \mathrm{H}_{2} \rightarrow \mathrm{CH}_{4}+\mathrm{H}_{2} \mathrm{O}, \\
& \mathrm{CO}+2 \mathrm{H}_{2} \rightarrow \mathrm{CH}_{3} \mathrm{OH} .
\end{aligned}
$$

Both the reactions correspond to the production of a polar species from nonpolar. It is then inferred from the results in Sec. IV that the equilibria are shifted to the product sides in the presence of solvent water. This qualitative insight is indeed supported by experimental observations. In hot water, the methanation reaction given by Eq. (15) was reported to be possible $e^{65}$ and the backward reaction of Eq. (16) was found to be prohibited. ${ }^{24}$ Actually, the chemistry of the synthesis gas is a subject of intensive research. ${ }^{66-68}$ A quantitative analysis will thus be needed for the hydrothermal chemistry of the synthesis gas in a comprehensive and systematic manner.

The equilibrium constant is of central importance in studying chemical reactions, although it is often difficult to determine in solution. The present paper has described a scheme to evaluate the equilibrium constant in solution from the standard free energies of formation in the absence of solvent and the solvation free energies of the reactive species. An equally important quantity in a chemical reaction is the rate constant. In order to estimate the rate constant, the transition state needs to specified with its solvation free energy. When water serves as a reaction medium, in particular, a water-assisted form is considered to greatly reduce the free energy of activation. ${ }^{41-43}$ Since the solvation free energy for a given structure of the transition state can be calculated without difficulty in the method of energy representation, the challenge is to determine the transition state structure. Our computational study of the hydrothermal chemistry of formaldehyde and formic acid will be completed with the freeenergy analysis of the transition state.

\section{ACKNOWLEDGMENTS}

This work was supported by the Grant-in-Aid for Scientific Research (Grant No. 15205004) from Japan Society for the Promotion of Science and by the Grant-in-Aid for Scientific Research on Priority Areas (Grant No. 15076205), the Grant-in-Aid for Creative Scientific Research (Grant No. 13NP0201), and the NAREGI (National Research Grid Initiative) Project from the Ministry of Education, Culture, Sports, Science, and Technology. The work was also benefited through the discussions with Dr. C. Wakai, Y. Nagai, K. Yoshida, and S. Morooka of Kyoto University. N.M. is further grateful to the Supercomputer Laboratory of Institute for Chemical Research, Kyoto University, for generous allocation of computation time.

\section{APPENDIX: THE DENSITY DEPENDENCE OF $\Delta \mu$ AND THE SOLUTE-SOLVENT RADIAL DISTRIBUTION FUNCTION}

The solution structure is typically described in terms of the radial distribution function. Especially, the solute-solvent radial distribution function characterizes the local density of the solvent around the solute relative to the bulk, and is directly related to the partial molar volumetric properties of the solute. ${ }^{69-74}$ Although the chemical equilibrium is solely governed by the chemical potential (free energy), the partial molar volumetric properties may be helpful to analyze or interpret the response of the equilibrium to the change in the density (or pressure). In this appendix, the density dependence of the solvation free energy $\Delta \mu$ of the solute is discussed in connection to the solute-solvent radial distribution function.

When the excess partial molar volume of the solute is $\Delta V$ and the solute-solvent radial distribution function is $\mathrm{g}(r)$ with the distance $r$, the derivative of $\beta \Delta \mu$ with respect to the (solvent) density $\rho$ is expressed as ${ }^{69-71}$

$$
\begin{aligned}
& \left(\frac{\partial(\beta \Delta \mu)}{\partial \rho}\right)_{T}=\frac{1}{\rho k_{B} T \kappa_{T}} \Delta V, \\
& \Delta V=4 \pi \int d r r^{2}[1-\mathrm{g}(r)],
\end{aligned}
$$

where $\kappa_{T}$ is the isothermal compressibility of the system and is equal to the one for the pure water in the present work. It should be noted that Eqs. (A1) and (A2) are exact for any of the site-site radial distribution functions between the solute and solvent. Although the microscopic information provided by the radial distribution function is different among the choices of the tagged sites in the solute and solvent, the thermodynamic information obtained through the integration 


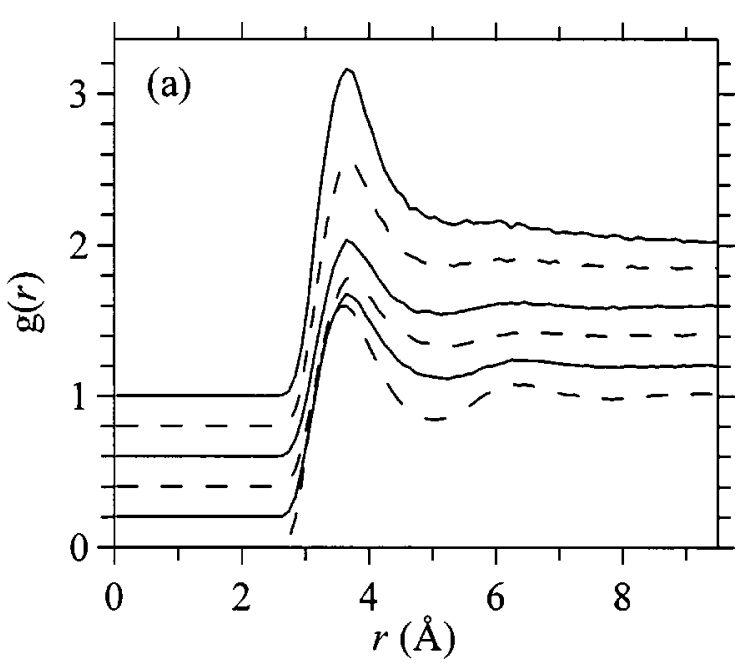

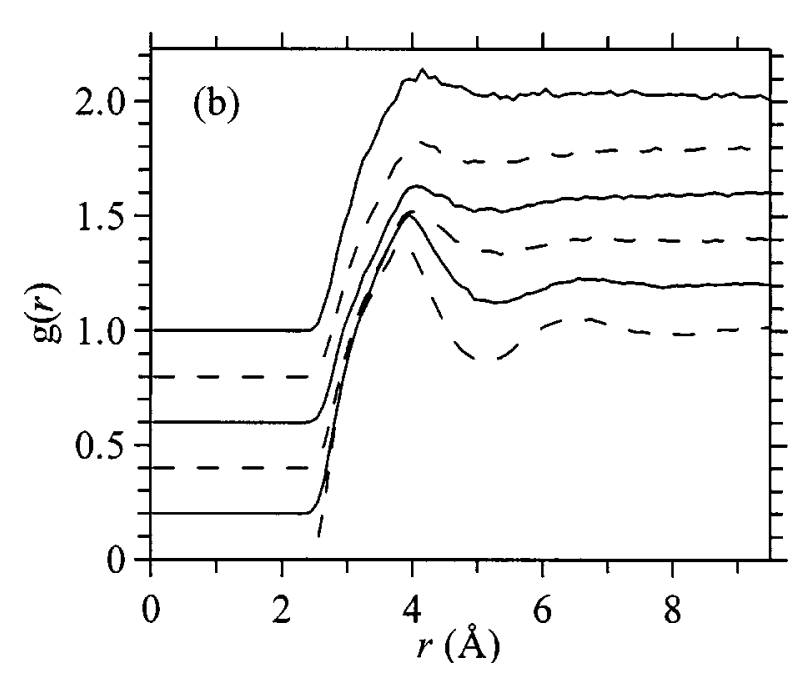

FIG. 10. The radial distribution function $\mathrm{g}(r)$ for (a) the solute $\mathrm{HCOOH}$ and (b) the solute $\mathrm{CO}_{2}$ as a function of the distance $r$ between the carbon site of the solute and the oxygen site of the solvent water. The temperature is fixed at $400{ }^{\circ} \mathrm{C}$. The solvent density is $0.10,0.20,0.40,0.60,0.80$, and $1.00 \mathrm{~g} / \mathrm{cm}^{3}$ from top to bottom with offsets of $1.0,0.8,0.6,0.4,0.2$, and 0 for $\mathrm{g}(r)$. The solid and dashed lines are used alternately from low to high density.

of Eq. (A2) is identical. $\rho k_{B} T \kappa_{T}$ is a dimensionless expression for the compressibility and approaches unity in the limit of zero density (ideal gas limit). Since $\rho k_{B} T \kappa_{T}$ is always positive, the sign of Eq. (A1) is coincident with that of $\Delta V$. According to Eq. (A2), the region of $r$ in which the local density is larger than in the bulk $[\mathrm{g}(r)>1]$ contributes negatively to $\Delta V$, and the region in which $\mathrm{g}(r)<1$ contributes positively. It should be noted that while $[\mathrm{g}(r)-1]$ at large $r$ becomes small in amplitude, its weight is enhanced due to the $4 \pi r^{2}$ factor in the integral.

The density dependence of $\mathrm{g}(r)$ at a fixed supercritical temperature of $400{ }^{\circ} \mathrm{C}$ is illustrated in Fig. 10 for typical cases of the solute $\mathrm{HCOOH}$ and $\mathrm{CO}_{2}$ by tagging the carbon site of the solute and the oxygen site of the solvent water. It is seen for both the solutes that the excluded volume region in which $\mathrm{g}(r) \simeq 0$ is insensitive to the density variation. The density effect appears outside the excluded volume region. In the low-density regime below $\sim 0.2 \mathrm{~g} / \mathrm{cm}^{3}, \mathrm{~g}(r)$ is not oscillatory and decays slowly to unity. The oscillatory behavior
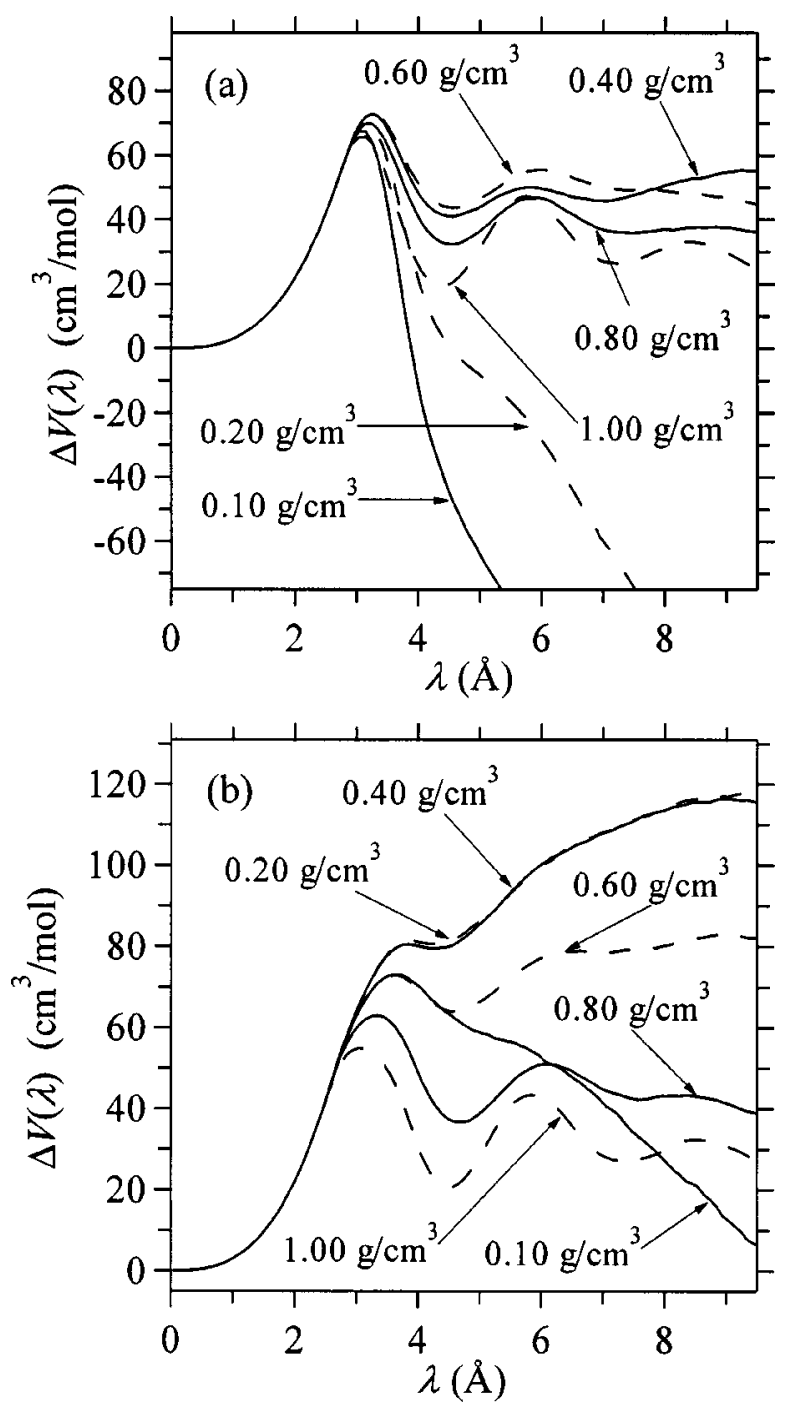

FIG. 11. The excess partial molar volume $\Delta V(\lambda)$ of (a) the solute $\mathrm{HCOOH}$ and (b) the solute $\mathrm{CO}_{2}$ as a function of the cutoff distance $\lambda$. The temperature is fixed at $400{ }^{\circ} \mathrm{C}$. The density in the figure refers to the solvent density. The solid and dashed lines are used alternately from low to high density.

develops at the medium-density regime, while the second peak is evident only in the high-density regime. The height of the first peak is not monotonic against the density variation. It reduces with the density elevation up to $0.4-0.6 \mathrm{~g} / \mathrm{cm}^{3}$, and increases in the higher-density regime.

To analyze the connection between the molecular-level information of the solvation structure and the thermodynamic observation of partial molar quantities, we developed the method of solvation shell analysis in previous papers. ${ }^{27,70,71,75}$ When the method is applied to $\Delta V$, the integral of Eq. (A2) is restricted to the region of $r<\lambda$ in the form of

$$
\Delta V(\lambda)=4 \pi \int_{0}^{\lambda} d r r^{2}[1-\mathrm{g}(r)],
$$

and the dependence of $\Delta V(\lambda)$ on the cutoff distance $\lambda$ is to be examined. ${ }^{70}$ The $\lambda$ dependence then clarifies the extent of localization of the response of the solution structure to solute insertion, in strong connection to the thermodynamic observable. Especially, it is possible to describe the relative weights 
of the contributions from the first and outer solvation shells. In the solvation shell analysis, the local view provided by the radial distribution function $\mathrm{g}(r)$ is transformed through the form of Eq. (A3) into the integral view directly related to a macroscopic observable.

In Fig. 11, we show $\Delta V(\lambda)$ of $\mathrm{HCOOH}$ and $\mathrm{CO}_{2}$ at $400{ }^{\circ} \mathrm{C}$ in correspondence to $\mathrm{g}(r)$ in Fig. 10. As typically illustrated in Ref. 70, the convergence of $\Delta V(\lambda)$ is not realized at the $\lambda$ corresponding to the first solvation shell and is to be attained only outside the $\lambda$ region of Fig. 11 . The excluded volume region [the region of $\mathrm{g}(r) \simeq 0$ ] always makes a positive contribution to $\Delta V$, and the solvation region [the region of $\mathrm{g}(r)>0$ ] determines the density dependence of $\Delta V$. In the low-density regime, the decay of $\mathrm{g}(r)$ against the $r$ increase is relatively slow in Fig. 10 and the outer portion of the solvation region needs to be taken into account in the evaluation of $\Delta V$. When the solute is $\mathrm{HCOOH}$, the attractive interaction between the solute and solvent is strong enough in the low-density regime that the excluded volume effect on $\Delta V$ is overwhelmed. When $\mathrm{CO}_{2}$ is concerned at low density, on the other hand, the competition between the attractive and repulsive interactions is not settled in the $\lambda$ region of Fig. 11 and the weak density dependence is observed for the solvation free energy $\Delta \mu$. In the medium- to high-density regime, an oscillatory profile is developed for $\mathrm{g}(r)$ and $\Delta V(\lambda)$ oscillates correspondingly as a function of the cutoff distance $\lambda$. The excluded volume effect is then seen in Fig. 11 to be only partially canceled, and $\Delta V$ is determined to be positive for both $\mathrm{HCOOH}$ and $\mathrm{CO}_{2}$. Actually, although the solvation shell structure is evident in the high-density regime, the contribution from the relatively high peak of the first shell does not lead to a negative $\Delta V$ in Fig. 11. According to Table I and Fig. 2, the density dependence of $\beta \Delta \mu$ is increasingly steep when the density is high. Figure 11 shows, in contrast, that $\Delta V$ is not a strong function of the density in the highdensity regime. The contrast is caused by the factor $\rho k_{B} T \kappa_{T}$ in Eq. (A1). Indeed, $\rho k_{B} T \kappa_{T}$ decreases rapidly against the density elevation in the medium- to high-density regime. 7,37

${ }^{1}$ Organic Synthesis in Water, edited by P. A. Grieco (Thomson Science, London, 1998).

${ }^{2}$ R. Breslow, Acc. Chem. Res. 24, 159 (1991).

${ }^{3}$ Water, A Comprehensive Treatise, edited by F. Franks (Plenum, New York, 1972), Vols. 1-7.

${ }^{4}$ R. W. Shaw, T. B. Brill, A. A. Clifford, C. A. Eckert, and E. U. Franck, Chem. Eng. News 69 (51), 26 (1991).

${ }^{5}$ M. Siskin and A. R. Katritzky, Science 254, 231 (1991).

${ }^{6}$ P. E. Savage, Chem. Rev. (Washington, D.C.) 99, 603 (1999).

${ }^{7}$ N. Matubayasi and M. Nakahara, J. Chem. Phys. 112, 8089 (2000).

${ }^{8}$ N. Akiya and P. E. Savage, Chem. Rev. (Washington, D.C.) 102, 2725 (2002).

${ }^{9}$ R. T. Morrison and R. N. Boyd, Organic Chemistry (Allyn \& Bacon, Boston, 2000).

${ }^{10}$ Y. Tsujino, C. Wakai, N. Matubayasi, and M. Nakahara, Chem. Lett. 1999, 287 (1999).

${ }^{11}$ D. Bröll, C. Kaul, A. Krämer, P. Krammer, T. Richter, M. Jung, H. Vogel, and P. Zehner, Angew. Chem., Int. Ed. 38, 2998 (1999).

${ }^{12}$ C. Wakai, S. Morooka, N. Matubayasi, and M. Nakahara, Chem. Lett. 33, 302 (2004).

${ }^{13}$ M. Osada, M. Watanabe, K. Sue, T. Adschiri, and K. Arai, J. Supercrit. Fluids 28, 219 (2004).

${ }^{14}$ Y. Nagai, C. Wakai, N. Matubayasi, and M. Nakahara, Chem. Lett. 32, 310 (2003).

${ }^{15}$ Y. Nagai, N. Matubayasi, and M. Nakahara, Chem. Lett. 33, 622 (2004).
${ }^{16}$ A. B. Bjerre and E. Sørensen, Ind. Eng. Chem. Res. 31, 1574 (1992).

${ }^{17}$ J. Yu and P. E. Savage, Ind. Eng. Chem. Res. 37, 2 (1998).

${ }^{18}$ P. G. Maiella and T. B. Brill, J. Phys. Chem. A 102, 5886 (1998).

${ }^{19}$ C. Wakai, K. Yoshida, Y. Tsujino, N. Matubayasi, and M. Nakahara, Chem. Lett. 33, 572 (2004).

${ }^{20}$ K. Yoshida, C. Wakai, N. Matubayasi, and M. Nakahara, J. Phys. Chem. A 108, 7479 (2004).

${ }^{21}$ Kirk-Othmer Encyclopedia of Chemical Technology, 4th ed., edited by J. I. Kroschwitz and M. Howe-Grant (Wiley, New York, 1991).

${ }^{22}$ W. M. Graven and F. J. Long, J. Am. Chem. Soc. 76, 2602 (1954).

${ }^{23}$ H. R. Holgate, P. A. Webley, J. W. Tester, and R. K. Helling, Energy Fuels 6, 586 (1992).

${ }^{24}$ Th. Hirth and E. U. Franck, Ber. Bunsenges. Phys. Chem. 97, 1091 (1993).

${ }^{25}$ S. F. Rice, R. R. Steeper, and J. D. Aiken, J. Phys. Chem. A 102, 2673 (1998).

${ }^{26}$ T. Sato, S. Kurosawa, R. L. Smith, Jr., T. Adschiri, and K. Arai, J. Supercrit. Fluids 29, 113 (2004).

${ }^{27}$ Since the backward reaction of Eq. (4) is called "hydration," we avoid the phrase hydration to denote the effect of intermolecular interaction between the solute and the solvent water. Instead, we use the phrase "solvation" to refer to the intermolecular interaction effects.

${ }^{28}$ N. Matubayasi and M. Nakahara, J. Chem. Phys. 117, 3605 (2002); 118, 2446 (2003)

${ }^{29}$ N. Matubayasi and M. Nakahara, J. Chem. Phys. 113, 6070 (2000).

${ }^{30}$ N. Matubayasi and M. Nakahara, J. Chem. Phys. 119, 9686 (2003).

${ }^{31}$ H. Takahashi, N. Matubayasi, M. Nakahara, and T. Nitta, J. Chem. Phys. 121, 3989 (2004).

${ }^{32}$ M. P. Allen and D. J. Tildesley, Computer Simulation of Liquids (Oxford University Press, Oxford, 1987).

${ }^{33}$ In our treatment, the contribution from the vibrational degrees of freedom is included in $E_{0}$ for each species. When the temperature is such that the molecule is in the ground state of vibration, $E_{0}$ is equal to the negative of the energy of dissociation to the component atoms. The correction due to the vibrational excitation is given by

$$
k_{B} T \sum \ln [1-\exp (-\beta h \nu)]
$$

within the harmonic approximation, where $h$ is the Planck constant, $\nu$ is the vibrational frequency, and the sum is taken over the vibrational degrees of freedom. The effect of the vibrational excitation is actually included in the equilibrium constant $K_{0}$ introduced by Eq. (14). However, the vibrational effect is $\sim k_{B} T$ for the molecules treated in the present work and does not affect our arguments even if it is neglected.

${ }^{34}$ With the equilibrium constant $K$ expressed as Eq. (10), the standard freeenergy change usually refers to $-k_{B} T \ln K$, not to $\left(\Delta E_{0}+\Delta W\right)$ in Eq. (10). Since $K$ is not a dimensionless quantity, it is always necessary to specify the unit of $K$ when the corresponding standard free-energy change is to be mentioned.

${ }^{35}$ When some of the reactive species are at finite concentration and are not coincident with the solvent species, $\Delta \mu_{i}$ (and possibly $E_{0 i}$ ) are functions also of the solute concentration. In this case, although Eq. (10) is a valid expression, $K$ depends on the solute concentration and cannot serve as an "equilibrium constant." When one of the reactive species is the solvent itself and is present in excess, on the other hand, its concentration (density) does not change in the course of reaction and the $\rho$ value in Eq. (10) is simply equal to the input density. Equation (10) is thus assured to be valid as an expression for the equilibrium constant when one of the reactive species is the solvent and the others are at infinite dilution.

${ }^{36}$ H. J. C. Berendsen, J. R. Grigera, and T. P. Straatsma, J. Phys. Chem. 91, 6269 (1987).

${ }^{37}$ The International Association for the Properties of Water and Steam, IAPWS Formulation 1995, Fredericia, Denmark, 1996.

${ }^{38}$ J. D. Madura and W. L. Jorgensen, J. Am. Chem. Soc. 108, 2517 (1986).

${ }^{39}$ P. Jedlovszky and L. Turi, J. Phys. Chem. B 101, 5429 (1997).

${ }^{40}$ P. Mináry, P. Jedlovszky, M. Mezei, and L. Turi, J. Phys. Chem. B 104, 8287 (2000).

${ }^{41}$ C. F. Melius, N. E. Bergan, and J. E. Shepherd, Proceedings of the TwentyThird Symposium (International) on Combustion (The Combustion Institute, Pittsburgh, PA, 1990), p. 217.

${ }^{42}$ N. Akiya and P. E. Savage, AIChE J. 44, 405 (1998).

${ }^{43}$ T. Yagasaki, S. Saito, and I. Ohmine, J. Chem. Phys. 117, 7631 (2002).

${ }^{44}$ W. L. Jorgensen, J. Phys. Chem. 90, 1276 (1986). 
${ }^{45}$ T. A. Bruce, Phys. Rev. B 5, 4170 (1972).

${ }^{46}$ Kagaku Binran (Dictionary of Chemistry), 5th ed., edited by Chemical Society of Japan (Maruzen, Tokyo, 2004) (in Japanese) (revised edition).

${ }^{47}$ J. G. Harris and K. H. Yung, J. Phys. Chem. 99, 12021 (1995).

${ }^{48}$ A. D. Buckingham and R. L. Disch, Proc. R. Soc. London, Ser. A 273, 275 (1963).

${ }^{49}$ J. P. Hansen and I. R. McDonald, Theory of Simple Liquids, 2nd ed. (Academic, London, 1986).

${ }^{50}$ Since the dipole moment is small, $\mathrm{CO}$ is categorized as nonpolar.

${ }^{51}$ When the solvent density is low enough, the density derivative of $\beta \Delta \mu$ at constant temperature has the same sign as $\int d \mathbf{x}\{1-\exp [-\beta v(\mathbf{x})]\}$, where $\mathbf{x}$ is the solvent configuration relative to the solute and $v(\mathbf{x})$ is the solutesolvent interaction. The excluded volume region, where $v(\mathbf{x})$ is very large, always makes a positive contribution to the density derivative of $\beta \Delta \mu$. The density derivative is reduced by the attractive (negative) portion of $v(\mathbf{x})$, and is not necessarily positive at low temperatures. In Table I and Fig. 2, the density derivative of $\beta \Delta \mu$ of a polar solute is negative at $400{ }^{\circ} \mathrm{C}$ in the low-density region. In other words, $400{ }^{\circ} \mathrm{C}$ is not high enough in the sense that the contribution from the attractive part of the solute-solvent interaction is not overwhelmed by that from the repulsive part. It should be noted that the attractive contribution is more sensitive to the temperature variation than the repulsive contribution and that the repulsive contribution dominates at a high enough temperature.

${ }^{52}$ It is a rather rough statement that the density corresponding to the minimum $\beta \Delta \mu$ is larger when the solute-water interaction is stronger. Indeed, the interaction strength is not a uniquely definable concept, but expresses the "intuitive feeling" about the molecule.

${ }^{53}$ H. Sato, N. Matubayasi, M. Nakahara, and F. Hirata, Chem. Phys. Lett. 323, 257 (2000).

${ }^{54}$ R. Crovetto, R. Fernández-Prini, and M. L. Japas, J. Chem. Phys. 76, 1077 (1982).

${ }^{55}$ R. Fernandez-Prini, R. Crovetto, M. L. Japas, and D. Laria, Acc. Chem. Res. 18, 207 (1985).

${ }^{56}$ R. Fernández Prini and R. Crovetto, J. Phys. Chem. Ref. Data 18, 1231 (1989).

${ }^{57}$ R. Fernández-Prini, J. L. Alvarez, and A. H. Harvey, J. Phys. Chem. Ref. Data 32, 903 (2003).

${ }^{58}$ B. Guillot and Y. Guissani, J. Chem. Phys. 99, 8075 (1993).

${ }^{59}$ In the present treatment, the Henry's constant is given by $\rho k_{B} T \exp (\beta \Delta \mu)$, where $\rho$ is the density of the solvent water.

${ }^{60}$ According to Ref. 57, the experimentally determined value of $\beta \Delta \mu$ is 3.7, $3.9,3.6,3.2,2.8,2.3$, and 1.5 for $\mathrm{CO}$ at $25,100,150,200,250,300$, and $350{ }^{\circ} \mathrm{C}$ on the liquid branch of the saturation curve, respectively. Similarly, the experimental value of $\beta \Delta \mu$ is $0.2,1.1,1.3,1.2,1.0,0.7$, and 0.4 for $\mathrm{CO}_{2}$ and 4.0, 3.8, 3.4, 3.0, 2.5, 1.9, and 1.2 for $\mathrm{H}_{2}$. A good agreement is thus observed between these experimental values and the corresponding calculated values in Table I, while the calculated values are more unfavorable by $\sim 1 \mathrm{kcal} / \mathrm{mol}$ for $\mathrm{CO}_{2}$.
${ }^{61}$ NASA Glenn thermodynamic database, URL: http://cea.grc.nasa.gov

${ }^{62}$ As done in Ref. 61, the standard free energy of formation is typically listed at the pressure of $1 \mathrm{~atm}$ or 1 bar. The pressure equilibrium constant $K_{P}$ is then obtained through a chemical equation and is related to the equilibrium constant $K_{0}$ of Eq. (14) by

$$
\ln K_{0}=\ln K_{P}-\left(\sum_{i \text { :product }} \nu_{i}-\sum_{i \text { :reactant }} \nu_{i}\right) \ln \left(k_{B} T\right)
$$

in the dilute gas condition.

${ }^{63}$ According to Ref. 24, $\beta \Delta W$ of the water-gas-shift reaction at a pressure of 400 bar is estimated to be $0.5-1.0$ and $1.3-1.7$ at temperatures of 500 and $600{ }^{\circ} \mathrm{C}$, respectively. The Arrhenius extrapolation of the data then leads to $\beta \Delta W \simeq 0$ at $400{ }^{\circ} \mathrm{C}$. The solvent density at the temperature of $400{ }^{\circ} \mathrm{C}$ needs to be treated with care, however, in such an extrapolation. At the pressure of $400 \mathrm{bar}$, the density is $0.52,0.18$, and $0.12 \mathrm{~g} / \mathrm{cm}^{3}$ at 400,500 , and $600{ }^{\circ} \mathrm{C}$, respectively (Ref. 37), and the variation is too large between 400 and $500{ }^{\circ} \mathrm{C}$ for an isobaric reference to the density in the extrapolation. A reasonable view is to refer to a density of $0.2-0.3 \mathrm{~g} / \mathrm{cm}^{3}$ when the extrapolation to $400{ }^{\circ} \mathrm{C}$ is performed. In this view, the extrapolated $\beta \Delta W$ at $400{ }^{\circ} \mathrm{C}$ is in good agreement with the corresponding $\beta \Delta W$ value given by Figs. 5 and 8 .

${ }^{64}$ According to Ref. $20, \ln K$ for the $\mathrm{HCOOH}$ decarbonylation is $\sim 4$ at $250{ }^{\circ} \mathrm{C}$ on the liquid branch of the saturation curve in our unit convention. This is in good agreement with the result shown in Fig. 7. The comparison is not attempted for the $\mathrm{HCOOH}$ decarboxylation, on the other hand, since the equilibrium is not achieved yet for the decarboxylation within the reaction time of Ref. 20. The experimental determination of the equilibrium constant for the $\mathrm{HCOOH}$ decarboxylation might be difficult due to a long reaction time required and a possible leakage of $\mathrm{H}_{2}$ through the quartz vessel used.

${ }^{65}$ T. Minowa and Z. Fang, J. Chem. Eng. Jpn. 31, 488 (1998).

${ }^{66}$ I. Wender, Fuel Process. Technol. 48, 189 (1996).

${ }^{67}$ C. B. Roberts and N. O. Elbashir, Fuel Process. Technol. 83, 1 (2003).

${ }^{68}$ M. Stelmachowski and L. Nowicki, Appl. Energy 74, 85 (2003).

${ }^{69}$ J. G. Kirkwood and F. P. Buff, J. Chem. Phys. 19, 774 (1951).

${ }^{70}$ N. Matubayasi and R. M. Levy, J. Phys. Chem. 100, 2681 (1996).

${ }^{71}$ N. Matubayasi, E. Gallicchio, and R. M. Levy, J. Chem. Phys. 109, 4864 (1998).

${ }^{72}$ T. Imai, M. Kinoshita, and F. Hirata, J. Chem. Phys. 112, 9469 (2000).

${ }^{73}$ T. Imai, H. Nomura, M. Kinoshita, and F. Hirata, J. Phys. Chem. B 106, 7308 (2002).

${ }^{74}$ T. Imai and F. Hirata, J. Chem. Phys. 119, 5623 (2003).

${ }^{75}$ N. Matubayasi, L. H. Reed, and R. M. Levy, J. Phys. Chem. 98, 10640 (1994). 